\title{
Avaliação perioperatória de indivíduos em quimioterapia com necessidade de intervenção cirúrgica odontológica
}

\author{
Perioperative evaluation of chemotherapy patients requiring surgical intervention
}

\author{
Bruno Gomes Duarte ${ }^{1}$, Aloizio Premoli Maciel ${ }^{2}$, Eduardo Sanches Gonçales ${ }^{3}$, Paulo Sérgio da Silva Santos ${ }^{3}$
}

\section{Resumo}

A quimioterapia é uma modalidade de tratamento antineoplásico que além de promover sua ação sob as células cancerígenas, exerce também efeito nas células do sistema hematopoiético causando alterações hematológicas. O conhecimento sobre o momento em que esses efeitos colaterais estão presentes e suas implicações clínicas são necessários quando os indivíduos em quimioterapia necessitam de intervenção cirúrgica odontológica. O objetivo deste artigo é realizar uma revisão sobre os tópicos referentes as alterações no hemograma no preparo cirúrgico bucal dos indivíduos submetidos a QT em estágio de Nadir. As considerações finais dessa revisão apontam que a cirurgias orais em indivíduos sob quimioterapia são exequíveis, desde que se indique corretamente o procedimento cirúrgico através da interpretação do hemograma completo e da avaliação do momento da intervenção cirúrgica de acordo com o intervalo do ciclo de quimioterapia, a fim de evitar procedimentos cirúrgicos durante o estágio de Nadir.

Descritores: Contagem de Células Sanguíneas, Cirurgia Bucal, Anemia, Quimioterapia, Trombocitopenia. Leucopenia

1. Mestrando na área de Cirurgia e Traumatologia Buco-Maxilo-Facial, Departamento de Cirurgia, Estomatologia, Patologia e Radiologia, Faculdade de Odontologia de Bauru - Universidade São Paulo (FOB-USP)

2. Doutorando na área de Estomatologia, Departamento de Cirurgia, Estomatologia, Patologia e Radiologia, Faculdade de Odontologia de Bauru - Universidade São Paulo (FOB-USP)

3. Professor Associado do Departamento de Cirurgia, Estomatologia, Patologia e Radiologia, Faculdade de Odontologia de Bauru - Universidade São Paulo (FOB-USP)

Trabalho realizado: Universidade de São Paulo. Faculdade de Odontologia de Bauru. Departamento de Cirurgia Estomatologia, Patologia e Radiologia

Endereço para correspondência: Paulo Sérgio da Silva Santos. Faculdade de Odontologia de Bauru, Universidade de São Paulo (FOB-USP). Departamento de Cirurgia, Estomatologia, Patologia e Radiologia. Al. Otávio Pinheiro Brizola, 9-75. CEP: 17017901. Telefone: +55 14 32358258.E-mail:psss@usp.br

\begin{abstract}
Chemotherapy is an antineoplastic treatment modality that in addition to promoting its action under the cancer cells, also exerts an effect on the cells of the hematopoietic system causing haematological alterations. Knowledge about the time at which chemotherapy side effects are present and its clinical implications are needed when individuals undergoing chemotherapy that require dental surgical intervention. The objective of the present article is to make a review of literature about the chemotherapy effects on the blood cell count in individual that require dental surgical intervention during Nadir. After this review of literature, we conclude that minor oral surgeries can be realized in patients undergoing chemotherapy treatment, if the dentist can indicate the surgical procedure through the hemogram and surgical intervention evaluation are according to the interval between courses of chemotherapy cycles, with intention to avoiding the surgical procedure during nadir.
\end{abstract}

Keywords: Blood Cell Count; Surgery, Oral; Anemia; Chemotherapy, Thrombocytopenia. Leucopenia

\section{Introdução}

A quimioterapia (QT) é uma modalidade de terapia antineoplásica dentre outros tipos existentes para o tratamento de indivíduos com câncer ${ }^{(1)}$. Atua em diferentes fases do ciclo celular majoritariamente de forma inespecífica para as células malignas, portanto exerce ação sobre as células com rápida proliferação, ocasionando destruição tanto de células neoplásicas quanto células benignas ${ }^{(1-2)}$. As células hematopoiéticas são muito afetadas pela QT, o que classifica esse tipo de tratamento como mielotóxico ou mielossupressor ${ }^{(3-4)}$.

A ação mielossupressora dos quimioterápicos resulta na incapacidade da medula óssea de repor os elementos figurados do sangue, predispondo a quadros de anemia, leucopenia, neutropenia e trombocitopenia. Desta forma, há necessidade de avaliação pré-operatória direcionada aos casos com indicação cirúrgica odontológica ${ }^{(1,3-4)}$, a qual consiste em anamnese, exame físico e interpretação dos exames 
laboratoriais, com foco nos efeitos adversos da QT, os quais podem estar relacionados com aumento da morbidade e mortalidade ${ }^{(1)}$.

Apesar da QT resultar em alterações nos exames laboratoriais ${ }^{(5)}$, existem poucos estudos que abordam a importância desses exames na avaliação perioperatória para os casos em que há a necessidade de intervenção cirúrgica durante a QT. Dessa forma o presente artigo tem como objetivo realizar uma revisão sobre os tópicos referentes as alterações no perfil de coagulação e hemograma no preparo cirúrgico bucal dos indivíduos submetidos a QT em estágio de Nadir.

\section{Revisão de Literatura}

\section{a) Nadir}

O termo "Nadir", vem da astronomia, que significa o ponto mais baixo no horizonte ${ }^{(6)}$. A mielossupressão decorrente da QT ocorre entre as primeiras 48 a 72 horas após a infusão do quimioterápico no organismo, neste período as células da medula óssea interrompem seu processo de divisão, ocorrendo uma grande queda da contagem dessas células hematopoiéticas circulantes no sangue periférico por um período de 10 a 14 dias, sendo esse período conhecido como $\operatorname{Nadir}^{(4,6)}$. A recuperação medular ocorre entre 15 a 21 dias após o término do ciclo de QT, o que resulta na normalização dos valores do hemograma ${ }^{(4)}$.

Esse conceito é extremamente importante para os profissionais de saúde que tratam indivíduos com câncer, uma vez que durante o Nadir esses pacientes podem estar mielossuprimidos e consequentemente mais susceptíveis a infecções e condições de hemorragia primária, levando a necessidade de internação e tratamento em ambiente hospitalar além de possível atraso no tratamento antineoplásico ${ }^{(7)}$. Embora esses conceitos estejam claramente sedimentados em livros de clínica médica, pouco se conhece na área de cirurgia oral sobre a importância do Nadir para o manejo odontológico de pacientes sob QT.

O entendimento do Nadir deve levar em consideração à sua variabilidade dentre os quimioterápicos e as possíveis complicações como a neutropenia febril. Durante este período, tratamentos odontológicos invasivos muitas vezes necessitam ser adiados até os parâmetros laboratoriais do hemograma se estabilizarem, o que pode resultar no tratamento tardio e evolução do quadro clínico da doença oncológica, estando indicado somente as intervenções cirúrgicas para os casos de infecções de origem bucal com risco de rápida disseminação mesmo durante o Nadir. A determinação da conduta odontológica para procedimentos cirúrgicos eletivos ou de urgência dependem da gravidade da doença bucal a ser tratada e da mie- lossupressão presente no momento da intervenção, sendo esta condição passível de análise através de exames sanguíneos como o hemograma completo e perfil de coagulação.

\section{b) Alterações no hemograma e mielossupressão}

O hemograma consiste na avaliação qualitativa e quantitativa das células vermelhas, brancas e plaquetas do sangue $^{(5)}$. Esse exame é fundamental para a avaliação do grau de mielossupressão que o indivíduo se encontra a fim de determinar as condutas perioperatórias de cirurgias bucais.

A mielossupressão pode ser traduzida no hemograma por: anemia, diminuição do número de eritrócitos; hemoglobina e hematócrito; neutropenia, diminuição do número de neutrófilos; leucopenia, diminuição do número de leucócitos; trombocitopenia, diminuição do número de plaquetas; e a pancitopenia, que consiste na diminuição simultânea de e eritrócitos, leucócitos e plaquetas.

A anemia é definida como a "condição em que ocorre deficiência de células vermelhas, hemoglobina ou no volume total"(8), ocorrendo com frequência em virtude do câncer ou do tratamento antineoplásico ${ }^{(9)}$, resultando em anemia hemolítica para os indivíduos em uso de procarbazina e fludarabina ${ }^{(10)}$. É possível observar ao exame clínico a palidez de mucosas, esplenomegalia e caquexia em indivíduos anêmicos ${ }^{(9)}$, assim como sintomas de fraqueza generalizada, intolerância a exercícios físicos e dispnéia. Os casos mais graves podem evoluir com déficit na oxigenação tecidual e isquemia de orgãos terminais ${ }^{(10)}$.

Os procedimentos cirúrgicos bucais não deverão ser realizados nos indivíduos com anemia intensa, devido o alto potencial de sangramento secundário e capacidade de reparação tecidual diminuída. Valores de hemoglobina abaixo de $10 \mathrm{~g} / \mathrm{dL}$ afetam as interações entre os componentes figurados do sangue e diminuem a capacidade de uma coagulação eficiente ${ }^{(11)}$, havendo a necessidade de transfusões sanguíneas ${ }^{(9)}$, sendo possível observar deficiência de cicatrização nos indivíduos submetidos a procedimentos cirúrgi$\cos ^{(12)}$, lipotímia por deficiência de oxigenação cerebral, aumento do débito cardíaco ${ }^{(9,13)}$, alterações renais ${ }^{(14)}$, maior risco de metástase ${ }^{(9)}$ e mortalidade ${ }^{(9,13)}$.

A leucopenia é a diminuição do número absoluto de neutrófilos ${ }^{(1,13,15)}$ e em indivíduos saudáveis apresentam contagens entre 3.600 a 12.600 , porém valores entre 3.400 a 5.600 são considerados normais em indivíduos melanodermas ${ }^{(5,15)}$. A contagem de leucócitos pode variar nos indivíduos saudáveis de acordo com atividade física, estado emocional e momento do dia ${ }^{(13,15)}$.

Durante a QT a leucopenia representa uma complicação importante e frequentemente observada nos 
indivíduos que fazem uso de docetaxel e paclitaxel ${ }^{(10)}$ entre o sexto e o décimo dia após a $\mathrm{QT}^{(1)}$. Nesses casos existe aumento do risco de infecções decorrentes da flora endógena da região oral, podendo resultar em infecções graves ${ }^{(13,15)}$.

A avaliação quanto à presença de úlceras da mucosa oral e o comprometimento gengival por infecções (gengivite e/ou periodontite) pelo cirurgião-dentista durante o exame clínico em indivíduos com leucopenia é essencial para a identificação de potenciais vias de contaminação. Mesmo em casos com ausência de focos de infecção bucais, o indivíduo pode evoluir com mielossupressão e consequentemente para quadros de infecções fúngicas, bacterianas e virais ${ }^{(1,16-17)}$.

Cirurgias orais em indivíduos com leucopenia moderada podem ser realizadas sem profilaxia antibiótica. Já os casos com leucopenia grave necessitam a avaliação do potencial de infecção bucal pelo cirurgião-dentista para determinação do uso de antibióticos pré-operatórios ${ }^{(1)}$, porém ressaltamos a necessidade de estabelecimento de critérios claros, uma vez que isto ainda não é bem estabelecido na literatura.

Os indivíduos em tratamento antineoplásico apresentam maior risco de infecções primárias bucais, exacerbação de infecções crônicas ou a reativação de infecções virais e de evoluírem com bacteremia ${ }^{(18)}$ decorrentes de lesões pulpares, periodontais ou casos de pericoronarite ${ }^{(18)}$. A presença da mucosite oral decorrente de toxicidade do quimioterápico, resulta em solução de continuidade proporcionada pelas úlceras, tornando-se propício para a colonização e disseminação bacterianas ${ }^{(19)}$.

Nos casos de doença oncohematológica, as infecções fúngicas e virais como candidíase oral e a herpes simples podem ser mais prevalentes que mucosite oral durante e a $\mathrm{QT}^{(20)}$. A candidíase na região da orofaringe representa a infecção fúngica mais comum nos indivíduos com câncer ${ }^{(18)} \mathrm{e}$ ocorre principalmente por Candida albicans ${ }^{(18)}$, acometendo várias estruturas bucais sadias e doentes, como por exemplo, a colonização de sítios bucais com mucosite oral em 51\% dos $\operatorname{casos}^{(21)}$. Ambas infecções virais e fúngicas são mais frequentes no período de $\operatorname{Nadir}^{(18,22)}$, por isso há necessidade de avaliação odontológica de todas as estruturas bucais, não somente quanto a queixa do paciente ou da condição da região operatória durante esse período de tempo.

Frente a quadro clínico infeccioso o indivíduo é avaliado por meio de "leucograma e exame clínico. A leucopenia grave, deve ser considerada como fator de risco para o desenvolvimento de complicações infecciosas associadas à mortalidade. $\mathrm{O}$ quadro 1 mostra os valores de referência para essa avaliação, e para estas condições de risco é recomendada profilaxia antibiótica para a realização de procedimentos invasivos de indivíduos com neutropenia igual ou menor que 500 células $/ \mathrm{mm}^{(3)}$

AAssociação Americana de Cardiologia preconiza a profilaxia antibiótica em dose única de 30 a $60 \mathrm{~min}$ antes do procedimento cirúrgico oral para os pacientes imunossuprimidos, priorizando o uso de $2 \mathrm{~g}$ de Amoxicilina por via oral, e para os indivíduos alérgicos a este medicamento a utilização de Cefalexina $2 \mathrm{~g}$, Clindamicina 600mg ou Azitromicina 500mg. Caso que o indivíduo que não consiga tolerar a via oral, a indicação de uso consiste de Ampicilina, Ceftriaxona ou Cefazolina endovenoso ${ }^{(23)}$.

Os indivíduos pré-QT devem passar por avaliação odontológica minuciosa, com o objetivo de identificar a presença de dentes com cáries extensas ou mobilidade, doença periodontal e outros focos de infecção a fim de prevenir quadros de infecção mais graves como sepse. Algumas medidas que contribuem na diminuição dos índices de infecção e o número de microorganismos patógenos é a utilização de soluções com Gluconato de Clorexidina 0,12\% aquosa associada a uma escovação dentária orientada. Em virtude da possibilidade de traumas decorrentes da escovação dentária, os autores preconizam utilização de escovas macias (por exemplo: Curaprox - Ultrasoft ${ }^{\circledR}, \mathrm{TePe}-$ Special Care ${ }^{\circledR}$ ) para diminuir os traumas na mucosa oral e gengiva durante o período de mucosite oral. Cabe salientar que os pacientes devem ser orientados também com relação a manutenção da higiene da língua, evitando-se quadros de saburra lingual que contém acúmulo de bactérias e fungos.

A trombocitopenia consiste na diminuição do número de plaquetas, resultando em aumento do risco de sangramento ${ }^{(5)}$. Ao exame clínico é possível observar epistaxe, sangramento gengival, menorragia, petéquias, hematomas ${ }^{(10,24)}$ e hemorragias, podendo representar risco de mortalidade a estes indivíduos ${ }^{(10)}$ e necessidade de transfusões de plaquetas ${ }^{(10)}$. Durante a QT a sua ocorrência é cíclica, ocorrendo ao redor do $14^{\circ}$ dia da administração do quimioterápico seguido por rápido período de melhora( ${ }^{(3)}$, estando relacionado com o uso de oxiplatina, fludarabina e rituximab ${ }^{(24)}$.

O valor mínimo para contagem de plaquetas é de $150.000 / \mathrm{mm}^{(5,24)}$, porém não se observa sangramento anormal em procedimentos cirúrgicos com contagem de plaquetas maior ou igual a $50.000 / \mathrm{mm}^{(5,24)}$, o que permite a realização de cirurgias bucais com critérios de extensão e da habilidade no uso de hemostáticos locais.

\section{c) Cuidados pré, trans e pós-cirúrgicos}

Os exames laboratoriais servem como referência para as condutas a serem adotadas nos tempos cirúrgicos em procedimentos orais menores. A literatura apresenta poucos estudos que correlacionem as alterações laboratoriais com as condutas a serem ado- 
Quadro 1

\begin{tabular}{|c|c|c|}
\hline Risco cirúrgico & Valores laboratoriais & Complicações \\
\hline Baixo risco & $\begin{array}{l}\text { ANC: } 1000-1500 \text { células } / \mathrm{mm}^{3} \\
\text { PT: > 100.000 unidade }\end{array}$ & Neutropenia moderada \\
\hline Risco moderado & $\begin{array}{l}\text { ANC: } 500 \text { - } 1000 \text { células } / \mathrm{mm}^{3} \\
\text { Pt: 50-100.000 unidade }\end{array}$ & Risco de infecção bacteriana. \\
\hline $\begin{array}{l}\text { Risco alto } \\
\text { Agranulocitose }\end{array}$ & $\begin{array}{l}\text { ANC: } 500-200 \text { células } / \mathrm{mm}^{3} \\
\text { ANC }<200 \text { unidade } \\
\text { Pt: }<50.000 \text { unidade } / \mathrm{mm}^{3} \\
\mathrm{Hb}:<10 \mathrm{~g} / \mathrm{dL}\end{array}$ & $\begin{array}{l}\text { Risco de infecção bacteriana. Necessidade ATB profilático. } \\
\text { Risco de sangramento significativo. } \\
\text { Risco de complicações cardiovasculares. }\end{array}$ \\
\hline
\end{tabular}

ANC: Contagem total de neutrófilos; Hb: hemoglobina; Pt: plaquetas; ATB: antibiótico ${ }^{(1,15,28,31)}$.

tadas. No momento da avaliação pré-operatória os 3 parâmetros do hemograma (eritrograma, leucograma e plaquetograma) são avaliados para determinar o risco cirúrgico para cirurgias ambulatoriais em indivíduos sob QT (Quadro 1).

Em indivíduos com doenças onco-hematológicas, as principais indicações cirúrgicas são o abscesso dentário, cáries extensas e doença periodontal avançada. Um estudo mostrou que a necessidade de profilaxia antibiótica foi necessária em somente 26 indivíduos de 69 submetidos à exodontias, e complicações foram observadas em 09 indivíduos, resultando em atraso do tratamento oncológico, hemorragia, necessidade de transfusão de plaquetas e internação hospitalar. Apenas um caso de dor prolongada foi observada e nenhum caso de infecção pós-operatória ${ }^{(25)}$.

Um dos princípios fundamentais no tratamento de indivíduos com alterações onco-hematológicos, consiste em minizar ou mesmo evitar as possíveis complicações cirúrgicas. O diagnóstico e a prevenção de complicações bucais devem ser realizados antes do início da QT, principalmente a resolução de focos de infecção a fim de minimizar a necessidade de procedimentos invasivos durante o estágio de Nadir, evitando a exacerbação e complicações de infecções odontogênicas durante a mielossupressão.

Salienta-se a necessidade de lembrar que alguns fármacos, podem resultar em neutropenia como os antibióticos beta-lactâmicos, clotrimazol, anti-tireoideanos, ticlopidina, neurolépticos, anti-epiléticos, anti-inflamatórios não esteroidais ${ }^{(15,26)}$ e a clindamicina ${ }^{(27)}$.

As cirurgias orais menores podem ser realizadas com segurança em indivíduos com contagem de plaquetas $\geq 30.000 / \mu \mathrm{L}^{(28)}$ com possibilidade de intervenções cirúrgicas em contagem de plaquetas igual a $20.000 / \mu \mathrm{L}^{(29)}$. Os indivíduos que estão classificados nos grupos de risco baixo ou moderado para a ocorrência de hemorragia (quadro 1) podem ser manejados por meio de adequada sutura associada ou não a agentes hemostáticos locais, como por exemplo: celulose oxidada (Surgicel ${ }^{\circledR}$, Ethicon, Somerville, NJ; ActCel Coreva, Westlake Village, CA), espumas de gelatina $\left(\right.$ Gelfoam ${ }^{\circledR}$, Pfizer, New York, NY; Surgifoam ${ }^{\circledR}$, Ethicon, Somerville, NJ), ostene (Ceremed Inc., Los Angeles, CA), colágeno microfibrilar (Avitene Davol, Warwick, Reino Unido; Instat Ethicon 360, Somerville, $\mathrm{NJ}$ ), ácido tânico, ácido epsilonaminocapróico, ácido tranexâmico e cola de fibrina desde que não haja infecção local instalada.

Como opções terapêuticas em relação ao manejo pré-operatório dos indivíduos que evoluem com neutropenia, podemos citar o uso de alguns antimicrobianos: meropenem, cefazolina, clindamicina, cefepime, ampicilina com sulbactam e a vancomicina ${ }^{(29)}$. No entanto, não existe clareza na literatura sobre a escolha do medicamento ou da melhor indicação quanto o uso profilático ou terapêutico. A antibioticoterapia preferencialmente deve ser mantida no período pós-operatório por pelo menos14 dias seguida de avaliação do quadro infeccioso.

\section{Considerações finais}

Cirurgias orais menores em indivíduos sob quimioterapia são exequíveis, desde que haja a indicação correta para o procedimento cirúrgico através da interpretação do hemograma completo e da avaliação do momento de realização da intervenção cirúrgica de acordo com o intervalo do ciclo de quimioterapia, a fim de evitar procedimentos cirúrgicos durante o estágio de Nadir.

\section{Referências}

1. Ogle OE, Saker M. Perioperative considerations of the patient on cancer chemotherapy. Oral Maxillofacial Surg Clin North Am. 2006; 18(2):185-93.

2. Lima MFS, Minetto RC. Conhecimento de pacientes onco-hematológicos em tratamento quimioterápico sobre os cuidados 
para prevenção de infecções. Comun Ciênc Saúde. 2014; 25(1): $35-44$.

3. Vadhan-Raj S. Management of chemotherapy-induced thrombocytopenia: current status of thrombopoietic agents. Semin Hematol. 2009; 46(1 Suppl 2):S26-S32.

4. Ávila FF, Soares MAO, Silva SR. Perfil hematológico e bioquímico sérico de pacientes submetidas à quimioterapia antineoplásica. Rev Enferm Atenção Saúde. 2013; 2(2):32-45.

5. Santos PSS, Soares-Júnior LAV. Medicina bucal: a prática da odontologia hospitalar. São Paulo: Santos, 2012. 315p.

6. Patussi C, Sassi LM, Munhoz EC, Zanicotti RT, Schussel JL. Clinical assessment of oral mucositis and candidiasis compare to chemotherapic nadir in transplanted patients. Braz Oral Res. 2014; 28(1):1-7.

7. Jenkins P, Freeman S. Pretreatment haematological laboratory values predict for excessive myelosuppression in patients receiving adjuvant FEC chemotherapy for breast cancer. Ann Oncol. 2004; 20(1):34-40

8. Bryan LJ, Zakai NA. Why is my patient anemic? Hematol Oncol Clin North Am. 2012; 26(2):205-30.

9. Molitermo AR, Spivak JL. Anemia of cancer. Hematol Oncol Clin North Am. 1996; 10: 345-63.

10. Livshits Z, Rao RB, Smith SW. An approach to chemotherapyassociated toxicity. Emerg Med Clin North Am. 2014; 32(1):167203.

11. Greenberg MS, Glick M. Medicina oral de Burket: diagnóstico e tratamento. $10^{a}$. ed. São Paulo: Santos; 2008. 677p.

12. Mendonça RJ, Coutinho-Netto J. Aspectos celulares da cicatrização. An Bras Dermatol. 2009; 84(3):257-62.

13. Boxer L, Dale DC. Neutropenia: causes and consequences. Semin Hematol. 2002; 39(2):75-81.

14. Scholl M, Petersen LC, Oliveira TMC, Santos-Júnior CAN, Ferreira KB, Vargas-Neto JC. Anemia na insuficiência cardíaca e sua interação com a função renal. Rev SOCERJ. 2008; 21(6):365-71.

15. Reagan JL, Castillo JJ. Why is my patient neutropenic?. Hematol Oncol Clin Norht Am. 2012; 26(2):253-66.

16. Albuquerque RA, Morais VLL, Sobral APV. Protocolo de atendimento odontológico a pacientes oncológicos pediátricos - revisão da literatura. Rev Odontol UNESP. 2007; 36(3): 275-80.

17. Demian NM, Shum JW, Kessel IL, Eid A. Oral surgery in patients undergoing chemoradiation therapy. Oral Maxillofac Surg Clin North Am. 2014; 26(2):193-207.

18. Lerman MA, Laudenbach J, Marty FM, Baden LR, Treister NS. Management of oral infections in cancer patients. Dent Clin North Am. 2008; 52(1):129-53.

19. Bardellini E, Amadori F, Schumacher RF, Foresti I. Majorana A. A new emerging oral infection: Raoultella planticola in a boy with haematological malignancy. Eur Arch Paediatr Dent. 2017; 18(3):215-8.
20. Ramírez-Amador V, Zambrano JG, Anaya-Saavedra G, Zentella-Dehesa A, Irigoyen-Camacho E, Meráz-Cruz N, et al. TNF as marker of oral candidiasis, HSV infection, and mucositis onset during chemotherapy in leukemia patients. Oral Dis. 2017; 23(7):941-8.

21. Singh GK, Capoor MR, Nair D, Bhowmik KT. Spectrum of fungal infection in head and neck cancer patients on chemoradiotherapy. J Egypt Natl Canc Inst. 2017; 29(1):33-7.

22. Centurion BS, Garcia AS, Rubira-Bullen IRF, Santos PSS. Avaliação clínica e tratamento das complicações bucais pós quimioterapia e radioterapia. Rev Assoc Paul Cir Dent. 2012; 66(2):136-41.

23. Wilson W, Taubert KA, Gewitz M, Lockhart PB, Baddour LM, Levison M, et al. Prevention of Infective Endocarditis Guidelines From the American Heart Association A Guideline From the American Heart Association Rheumatic Fever, Endocarditis, and Kawasaki Disease Committee, Council on Cardiovascular Disease in the Young, and the Council on Clinical Cardiology, Council on Cardiovascular Surgery and Anesthesia, and the Quality of Care and Outcomes Research Interdisciplinary Working Group. Circulation. 2007; 116(15):1736-54.

24. Wong EY, Rose MG. Why does my patient have thrombocytopenia?. Hematol Oncol Clin North Am. 2012; 26(2):231-52.

25. Raut A, Huryn JM, Hwang FR, Zlotolow IM. Sequelae and complications related to dental extractions in patients with hematologic malignancies and the impact on medical outcome. Oral Surg Oral Med Oral Pathol Oral Radiol Endod. 2001; 92(1):49-55

26. Kim SK, Demetri GD. Chemotherapy and neutropenia. Hematol Oncol Clin North Am. 1996; 10(2):377-95.

27. Bubalo JS, Blasdel CS, Bearden DT. Neutropenia after single-dose clindamycin for dental prophylaxis. Pharmacotherapy. 2003; 23(1):101-3.

28. Henderson JM, Bergman S, Salama A, Koterwas G. Management of the oral and maxillofacial surgery patient with thrombocytopenia. J Oral Maxillofac Surg. 2001; 59(4):421-7.

29. Fillmore WJ, Leavitt BD, Arce K. Dental extraction in the neutropenic patient. J Oral Maxillofac Surg. 2014; 72(12):2386-93.

30. Guimarães GMMF, Ferreira-Junior O, Soares-Júnior LAV, Santos PSS. Manejo do paciente em terapia com anticoagulantes atuais em cirurgias orais e maxilofaciais. Rev Soc Cardiol Estado de São Paulo. 2016; 26(3):130-4.

31. Abubaker AO, Benson KJ. Oral and maxillofacial secrets. Philadelphia: Hanley \& Belfus Inc; 2001. 354p.

Trabalho recebido: 31/01/2018

Trabalho aprovado: 05/06/2018 